\title{
Changing Clinical and Therapeutic Trends in Tentorial Dural Arteriovenous Fistulas: A Systematic Review
}

\author{
D. Cannizzaro, W. Brinjikji, S. Rammos, M.H. Murad, and G. Lanzino
}

\begin{abstract}
BACKGROUND AND PURPOSE: Tentorial dural arteriovenous fistulas are characterized by a high hemorrhagic risk. We evaluated trends in outcomes and management of tentorial dural arteriovenous fistulas and performed a meta-analysis evaluating clinical and angiographic outcomes by treatment technique.
\end{abstract}

MATERIALS AND METHODS: We performed a comprehensive literature search for studies on surgical and endovascular treatment of tentorial dural arteriovenous fistulas. We compared the proportion of patients undergoing endovascular, surgical, and combined endovascular/surgical management; the proportion of patients presenting with ruptured tentorial dural arteriovenous fistulas; and proportion of patients with good neurologic outcome across 3 time periods: 1980-1995, 1996-2005, and 2006-2014. We performed a random-effects meta-analysis, evaluating the rates of occlusion, long-term good neurologic outcome, perioperative morbidity, and resolution of symptoms for the 3 treatment modalities.

RESULTS: Twenty-nine studies with 274 patients were included. The proportion of patients treated with surgical treatment alone decreased from $38.7 \%$ to $20.4 \%$ between $1980-1995$ and 2006-2014. The proportion of patients treated with endovascular therapy alone increased from $16.1 \%$ to $48.0 \%$. The proportion of patients presenting with ruptured tentorial dural arteriovenous fistulas decreased from $64.4 \%$ to $43.6 \%$. The rate of good neurologic outcome increased from $80.7 \%$ to $92.9 \%$. Complete occlusion rates were highest for patients receiving multimodality treatment (84.0\%; $95 \% \mathrm{Cl}, 72.0 \%-91.0 \%)$ and lowest for endovascular treatment $(71.0 \%$; $95 \% \mathrm{Cl}, 56.0 \%-83.0 \%$; $P<.01)$. Long-term good neurologic outcome was highest in the endovascular group (89.0\%; $95 \% \mathrm{Cl}, 80.0 \%-95.0 \%)$ and lowest for the surgical group $(73.0 \% ; 95 \% \mathrm{Cl}, 51.0 \%-87.0 \% ; P=.03)$.

CONCLUSIONS: Patients with tentorial dural arteriovenous fistulas are increasingly presenting with unruptured lesions, being treated endovascularly, and experiencing higher rates of good neurologic outcomes. Endovascular treatment was associated with superior neurologic outcomes but lower occlusion rates.

ABBREVIATIONS: DAVF = dural arteriovenous fistula; TDAVF $=$ tentorial dural arteriovenous fistula

ntracranial dural arteriovenous fistulas (DAVFs) are abnormal direct shunts between the dural arteries and dural veins. ${ }^{1}$ The shunt is located in the intracranial dura mater with venous drainage directed to the dural venous sinuses or cortical veins. Dural arteriovenous fistulas account for $10 \%-15 \%$ of all intracranial

\footnotetext{
Received January 29, 2015; accepted March 5.

From the Departments of Neurosurgery (D.C., G.L.) and Radiology (W.B.) and Center for Science of Healthcare Delivery (M.H.M.), Mayo Clinic, Rochester, Minnesota; and Department of Neurosurgery (S.R.), Arkansas Neuroscience Institute, Little Rock, Arkansas.

Please address correspondence to Waleed Brinjikji, MD, Department of Radiology, May Clinic, 200 1st St SW, Rochester, MN 55905; e-mail: brinjikji.waleed@mayo.edu; @WBrinjikji

Indicates article with supplemental on-line photo

Mvidence-Based Medicine Level 1.

http://dx.doi.org/10.3174/ajnr.A4394
}

vascular shunts. ${ }^{1,2}$ Tentorial dural arteriovenous fistulas (TDAVFs) constitute only $4 \%$ of DAVFs and are characterized by a high hemorrhagic risk. Because of this, these lesions are treated aggressively on diagnosis. ${ }^{3-5}$

Traditionally, surgical resection was the only treatment available for these lesions. However, endovascular embolization, either alone or in combination with surgery, is increasingly used. ${ }^{6}$ Stereotactic radiosurgery is also increasingly used as an adjunct to surgical and endovascular treatment. ${ }^{7}$ We performed a systematic review of the literature on surgical and endovascular treatment of TDAVFs from 1980 to 2014. The purpose of our study was the following: 1) to determine whether there was a shift from primarily surgical treatments to endovascular and multimodality treatment during this time period, 2) to determine whether the proportion of patients presenting with ruptured TDAVFs during this 
time period has changed, 3 ) to determine whether the rate of good neurologic outcome has changed, and 4) to evaluate clinical and angiographic outcomes in endovascular, surgical, and combined treatments by performing a random-effects meta-analysis.

\section{MATERIALS AND METHODS}

A comprehensive literature search of the data bases (PubMed, Ovid MEDLINE, and Ovid EMBASE) was designed and conducted by an experienced librarian with input from the authors. The key words "endovascular," "catheterization," "percutaneous," “embolization," “coil,” “cerebral veins," "intracranial," “arteriovenous fistula," "surgery," "DAVF," "resection," and "tentorial" were used in both "AND" and "OR" combinations. The search was limited to articles published from 1980 to June 2014 in the English language only. All studies reporting patients treated with surgery or endovascular therapy for TDAVFs were selected. Inclusion criteria were the following: 1) series of $>5$ patients, with available data on clinical and/or angiographic outcomes. Studies with fewer than 5 patients were included only if they were published before 1995 . Two reviewers selected the included studies. For each study, we extracted the following information: patient presentation (ruptured or unruptured), treatment technique (endovascular, surgical, or combined), long-term good neurologic outcome, long-term neurologic morbidity, angiographic occlusion status at last follow-up, resolution of symptoms for unruptured TDAVFs, and year of publication. Good neurologic outcome was defined as a modified Rankin Scale score of $\leq 2$. In cases in which a modified Rankin Scale score was not available, good neurologic outcome was determined if the study used terms such as "no morbidity" or "good recovery."

The primary objective of this study was to determine the clinical and angiographic outcomes of patients with endovascular, surgical, or multimodality treatment. The secondary objective was to determine trends in patient presentations, treatment type, and clinical outcome following treatment of TDAVFs. For this secondary objective, studies were divided into 3 periods: 1) studies published from 1995 and earlier, 2) studies published from 1996 to 2005, and 3) studies published from 2006 to 2014. For this secondary objective, we performed the following analyses: 1 ) the proportion of patients presenting with ruptured TDAVFs in each period; 2) the proportion of patients receiving endovascular, surgical, and combined treatments; and 3) the proportion of patients with good neurologic outcome.

\section{Study Quality}

We modified the Newcastle-Ottawa Quality Assessment Scale for case-control studies to assess the quality of the studies included in this meta-analysis. The Newcastle-Ottawa score is designed for use in case-control-type studies; however, because none of the studies included in our analysis were of this type, we assessed study quality on the basis of the following: 1) The study included all patients or consecutive patients versus a selected sample, 2) the angiographic and clinical follow-up was satisfactory, thus allowing ascertainment of all outcomes, 3) the case definition was adequate (ie, the location and presen- tation of the DAVF were clearly stated), 4) outcomes were clearly reported, and 5) the interventionalists/surgeons treating the patients were the same as those who assessed angiographic and clinical outcomes. High-quality studies were defined as those with $\geq 20$ patients, high rates of clinical follow-up with clearly reported outcomes, and independent assessment of angiographic and clinical outcomes.

\section{Statistical Analysis}

All included studies were noncomparative. We estimated from each cohort the cumulative incidence (event rate) and 95\% confidence interval for each outcome. Event rates for each intervention were pooled in a meta-analysis across studies by using the random-effects model. ${ }^{8}$ Anticipating heterogeneity between studies, we chose this model a priori because it incorporates within-study and between-study variances. We also extracted a $2 \times 2$ table for each studied outcome for interaction testing and calculated $P$ values for the comparisons between endovascular and surgical groups, surgical and multimodality groups, and endovascular and multimodality groups. Heterogeneity of treatment effect across studies was evaluated by using the $\mathrm{I}^{2}$ statistic. $^{9}$

\section{RESULTS}

\section{Literature Review}

Studies included in our review are summarized in Table 1. Our literature search yielded 986 articles; 879 articles were excluded after reading the abstract alone, with a total of 107 articles reviewed. Of these, 78 were excluded for either not including TDAVFs in their patient population or not reporting patient presentation or angiographic outcomes. In total, 29 studies with 274 patients met our inclusion criteria. The search results are summarized in Fig 1.

\section{Meta-Analysis Outcomes: All Patients}

Meta-analysis outcomes are summarized in Table 2. Forest plots are provided as On-line Figs 1-5. The rates of complete occlusion at last follow-up were $71.0 \%$ (95\% CI, 56.0\%-83.0\%) for the endovascular-only group, $81.0 \%$ (95\% CI, 61.0\%-92.0\%) for the surgery-only group, and $84.0 \%$ (95\% CI, 72.0\%-91.0\%) for the combined-treatment group. The combined-treatment group had a statistically significantly higher rate of complete occlusion $(P<.01)$. The rate of long-term neurologic morbidity was 5.0\% (95\% CI, 2.0\%-11.0\%) for the endovascular group, $8.0 \%(95 \% \mathrm{CI}, 2.0 \%-21.0 \%)$ for the surgery-only group, and 6.0\% (95\% CI, 2.0\%-15.0\%) for the combined-treatment group. There was no difference between groups in the rates of long-term neurologic morbidity. Perioperative morbidity occurred in $6.0 \%(95 \% \mathrm{CI}, 3.0 \%-13.0 \%)$ for the endovascular group, $18.0 \%$ ( $95 \% \mathrm{CI}, 8.0 \%-35.0 \%$ ) for the surgery-only group, and $14.0 \%(95 \% \mathrm{CI}, 8.0 \%-24.0 \%)$ for the combined-treatment group. Patients treated with endovascular therapy had significantly lower perioperative morbidity rates than those treated with surgery $(P=.03)$ and combined treatments $(P<.01)$. The long-term good neurologic outcome rates were $89.0 \%$ (95\% CI, $80.0 \%-95.0 \%$ ) for the endovascular group, $73.0 \%$ (95\% CI, $51.0 \%-87.0 \%$ for the surgical group, and $87.0 \%$ (95\% CI, $71.0 \%-94.0 \%)$ for the combined-treatment group. 
Table 1: Study characteristics

\begin{tabular}{|c|c|c|c|c|c|}
\hline Authors, Year & Treatment Type & Clinical/Angiographic Follow-Up (mo) & Rupture Status & No. of Patients & Risk of Bias \\
\hline Abud et al, $2011^{19}$ & SE & 6 & NA & 5 & Medium \\
\hline Bret et al, $1994^{20}$ & $\mathrm{~S}$ & 3 & $U$ & 1 & High \\
\hline Byrne and Garcia, $2013^{3}$ & SE & NA & RU & 13 & Medium \\
\hline Fardoun et al, $1981^{21}$ & $S$ & 12 & $\mathrm{R}$ & 1 & High \\
\hline Grisoli et al, $1984^{22}$ & S & 10 & RU & 4 & High \\
\hline Gross and Du, $2013^{23}$ & S & 25 & RU & 15 & Medium \\
\hline Hatano et al, $2013^{24}$ & $S$ & 6 & $U$ & 9 & Medium \\
\hline Huang et al, $2009^{25}$ & E & 13 & $\mathrm{RU}$ & 14 & Medium \\
\hline Ito et al, $1995^{26}$ & $\mathrm{~S}$ & 11 & $\mathrm{R}$ & 1 & High \\
\hline Kakarla et al, $2007^{27}$ & SC & 21.2 & $\mathrm{RU}$ & 21 & Medium \\
\hline Lawton et al, $2008^{28}$ & C & 50 & RU & 31 & Medium \\
\hline Lewis et al, $1994^{29}$ & $\mathrm{~S}$ & 12 & $\mathrm{RU}$ & 6 & Medium \\
\hline Lewis et al, $1997^{30}$ & $\mathrm{E}$ & 30 & $\mathrm{RU}$ & 9 & Medium \\
\hline Liu et al, $2014^{31}$ & $\mathrm{E}$ & 33 & $U$ & 26 & Medium \\
\hline Lucas Cde et al, $2005^{32}$ & SEC & 3 & NA & 20 & Medium \\
\hline Lucas et al, $1997^{33}$ & $\mathrm{EC}$ & NA & 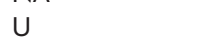 & 1 & High \\
\hline Natarajan et al, $2010^{34}$ & $\mathrm{EC}$ & 16.5 & $\mathrm{R}$ & 7 & Medium \\
\hline Ott et al, $1993^{35}$ & $\mathrm{E}$ & 16 & $U$ & 1 & High \\
\hline Pandey et al, $2011^{36}$ & $S$ & NA & NA & 6 & High \\
\hline Pierot et al, $1992^{37}$ & SEC & 1 & $u$ & 6 & High \\
\hline Piippo et al, $2013^{38}$ & SEC & NA & NA & 7 & High \\
\hline Puffer et al, $2012^{16}$ & $C$ & 4.6 & $\mathrm{RU}$ & 9 & Medium \\
\hline Rabinov et al, $2013^{39}$ & $E$ & NA & NA & 5 & Medium \\
\hline Tomak et al, $2003^{40}$ & SEC & 60 & $\mathrm{RU}$ & 22 & Medium \\
\hline van Lindert et al, $2000^{41}$ & $\mathrm{C}$ & 30 & $\mathrm{RU}$ & 5 & Medium \\
\hline van Rooij et al, $2006^{42}$ & $E$ & 3 & $\mathrm{RU}$ & 6 & Medium \\
\hline Wachter et al, $2011^{43}$ & SE & NA & NA & 9 & Medium \\
\hline Wajnberg et al, $2012^{44}$ & $\mathrm{E}$ & 15.5 & RU & 9 & Medium \\
\hline Zhou et al, $2007^{6}$ & SC & 30 & $\mathrm{RU}$ & 5 & Medium \\
\hline
\end{tabular}

Note:-S indicates surgery; E, endovascular; C, combined; R, ruptured; U, unruptured; NA, not available.

986 unique articles identified and screened

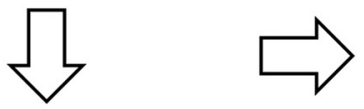

879 excluded based on abstract and title alone

107 full-text articles retrieved for more detailed evaluation and review

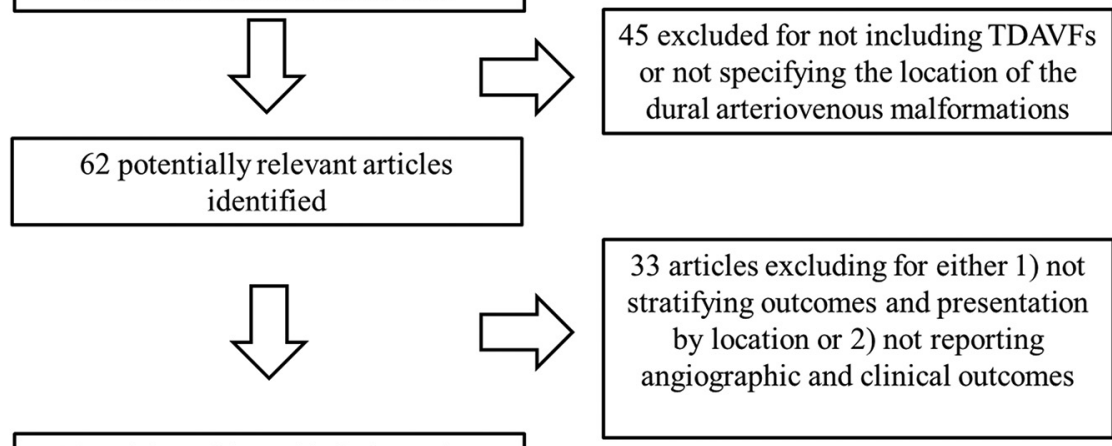

29 articles with usable information included in final study

FIG 1. Literature search flow chart.

Long-term good neurologic outcome rates were significantly higher in the endovascular group than in the surgery group $(P=.03)$.

Meta-Analysis Outcomes: Patients with Unruptured TDAVFs Meta-analysis outcomes are summarized in Table 2. Forest plots are provided as On-line Figs 1-5. The rates of complete occlusion at last follow-up were $76.0 \%$ (95\% CI, 54.0\%$89.0 \%)$ for the endovascular-only group, $89.0 \%$ (95\% CI, $69.0 \%-97.0 \%$ ) for the surgery-only group, and $78.0 \%$ (95\% CI, $53.0 \%-92.0 \%)$ for the combined-treatment group. The rates of long-term neurologic morbidity were $10.0 \%$ (95\% CI, $3.0 \%-27.0 \%)$ for the endovascular group, $11.0 \%$ (95\% CI, 3.0\%-31.0\%) for the surgery-only group, and $10.0 \%(95 \% \mathrm{CI}$, $3.0 \%-28.0 \%)$ for the combined-treatment group. Perioperative morbidity occurred in $10.0 \%$ (95\% CI, 3.0\%-27.0\%) for the endovascular group, $18.0 \%(95 \%$ CI, $5.0 \%-48.0 \%$ ) for the surgery-only group, and $21.0 \%$ (95\% CI, 8.0\%-46.0\%) for the combined-treatment group. The rates of resolution of symptoms were $82.0 \%$ (95\% CI, 59.0\%-94.0\%) for the endovascular group, $50.0 \% \quad(95 \% \mathrm{CI}$, $18.0 \%-82.0 \%)$ for the surgery-only group, and $73.0 \%$ (95\% CI, 37.0\%$92.0 \%)$ for the combined-treatment group. There were significantly higher rates of symptom resolution in the endovascular group compared with the surgical group $(P=.03)$. The long-term good neurologic outcome rates were $95.0 \%$ (95\% CI, 91.1\%-99.0\%) for the endovascular group, $56.0 \%$ (95\% CI, $20.0 \%-86.0 \%)$ for the surgical group, and $88.0 \%$ (95\% CI, 68.0\%-96.0\%) for the combined-treatment group, with significantly better outcomes in the endovascular compared with surgical group $(P<$ 


\begin{tabular}{|c|c|c|c|c|c|c|c|c|c|}
\hline & Endovascular & $I^{2}$ & Surgical & $1^{2}$ & Multimodality & $\mathrm{I}^{2}$ & $\begin{array}{c}P \text { Value } \\
\text { Evs S }\end{array}$ & $\begin{array}{l}P \text { Value } \\
\text { Evs M }\end{array}$ & $\begin{array}{l}P \text { Value } \\
\text { S vs M }\end{array}$ \\
\hline \multicolumn{10}{|l|}{ Ruptured and unruptured } \\
\hline Complete occlusion & $71.0(56.0-83.0)$ & 43 & $81.0(61.0-92.0)$ & 33 & $84.0(72.0-91.0)$ & 14 & .09 & $<.01$ & .63 \\
\hline $\begin{array}{l}\text { Long-term neurologic } \\
\text { morbidity }\end{array}$ & $5.0(2.0-11.0)$ & 0 & $8.0(2.0-21.0)$ & 0 & $6.0(2.0-15.0)$ & 0 & .66 & 1.00 & 1.00 \\
\hline Perioperative morbidity & $6.0(3.0-13.0)$ & 0 & $18.0(8.0-35.0)$ & 0 & $14.0(8.0-24.0)$ & 0 & .03 & $<.01$ & .76 \\
\hline $\begin{array}{l}\text { Good long-term neurologic } \\
\text { outcome }\end{array}$ & $89.0(80.0-95.0)$ & 0 & $73.0(51.0-87.0)$ & 21 & $87.0(71.0-94.0)$ & 0 & .03 & 1.00 & .07 \\
\hline \multicolumn{10}{|l|}{ Unruptured } \\
\hline Complete occlusion & $76.0(54.0-89.0)$ & 0 & $89.0(69.0-97.0)$ & 50 & $78.0(53.0-92.0)$ & 9 & .48 & .95 & .51 \\
\hline $\begin{array}{l}\text { Long-term neurologic } \\
\text { morbidity }\end{array}$ & $10.0(3.0-27.0)$ & 0 & $11.0(3.0-31.0)$ & 0 & $10.0(3.0-28.0)$ & 0 & .97 & 1.00 & .95 \\
\hline Perioperative morbidity & $10.0(3.0-27.0)$ & 0 & $18.0(5.0-48.0)$ & 0 & $21.0(8.0-46.0)$ & 0 & .25 & .45 & .70 \\
\hline $\begin{array}{l}\text { Good long-term neurologic } \\
\text { outcome }\end{array}$ & $95.0(91.0-99.0)$ & 55 & $56.0(20.0-86.0)$ & 0 & $87.0(66.0-96.0)$ & 0 & $<.01$ & .72 & $<.01$ \\
\hline $\begin{array}{l}\text { Resolution of symptoms } \\
\text { (unruptured only) }\end{array}$ & $82.0(59.0-94.0)$ & 0 & $50.0(18.0-82.0)$ & 48 & $73.0(37.0-92.0)$ & 0 & .03 & .57 & .42 \\
\hline \multicolumn{10}{|l|}{ Ruptured } \\
\hline Complete occlusion & $78.0(61.0-88.0)$ & 0 & $88.0(63.0-97.0)$ & 0 & $82.0(62.0-93.0)$ & 0 & .51 & .65 & .63 \\
\hline $\begin{array}{l}\text { Long-term neurologic } \\
\text { morbidity }\end{array}$ & $7.0(3.0-19.0)$ & 0 & $12.0(3.0-37.0)$ & 0 & $9.0(3.0-28.0)$ & 0 & .95 & .93 & .91 \\
\hline Perioperative morbidity & $7.0(3.0-19.0)$ & 0 & $20.0(6.0-49.0)$ & 0 & $21.0(10.0-38.0)$ & 0 & .14 & .02 & .76 \\
\hline $\begin{array}{l}\text { Good long-term neurologic } \\
\text { outcome }\end{array}$ & $87.0(71.0-94.0)$ & 0 & $73.0(44.0-90.0)$ & 0 & $88.0(68.0-96.0)$ & 0 & .44 & .84 & .22 \\
\hline
\end{tabular}

Note:-M indicates multimodality; S, surgery; E, endovascular.

$.01)$ and the combined-treatment compared with the surgical group $(P<.01)$.

\section{Meta-Analysis Outcomes: Patients with Ruptured TDAVFs} Meta-analysis outcomes are summarized in Table 2. Forest plots are provided as On-line Figs 1-5. The rates of complete occlusion at last follow-up were $78.0 \%$ (95\% CI, $61.0 \%-$ $88.0 \%$ ) for the endovascular-only group, $88.0 \%$ (95\% CI, $63.0 \%-97.0 \%)$ for the surgery-only group, and $82.0 \%(95 \%$ CI, $62.0 \%-93.0 \%)$ for the combined-treatment group. The rates of long-term neurologic morbidity were $7.0 \%(95 \%$ CI, 3.0\%-19.0\%) for the endovascular group, $12.0 \%$ (95\% CI, $3.0 \%-37.0 \%)$ for the surgery-only group, and $9.0 \%$ (95\% CI, $3.0 \%-28.0 \%)$ for the combined-treatment group. Perioperative morbidity occurred in $7.0 \%$ (95\% CI, 3.0\%-19.0\%) for the endovascular group, $20.0 \%$ (95\% CI, 6.0\%-49.0\%) for the surgery-only group, and $21.0 \%$ (95\% CI, 10.0\%-38.0\%) for the combined-treatment group. The long-term good neurologic outcome rates were $87.0 \%$ (95\% CI, 71.0\%-94.0\%) for the endovascular group, $73.0 \%$ (95\% CI, $44.0 \%-90.0 \%)$ for the surgical group, and $88.0 \%$ (95\% CI, 71.0\%-94.0\%) for the combined-treatment group. There were no significant differences among groups for any of the outcomes.

\section{Secondary Outcomes}

The proportion of patients treated with surgical treatment alone decreased from $38.7 \%$ to $20.4 \%$ between $1980-1995$ and $2006-$ 2014. The proportion of patients treated with endovascular therapy alone increased from $16.1 \%$ to $48.0 \%$ (Fig 2). The proportion of patients presenting with ruptured TDAVFs decreased from $64.4 \%$ to $43.6 \%$ (Fig 3). The rate of good neurologic outcome increased from $80.7 \%$ to $92.9 \%$ (Fig 4).

\section{DISCUSSION}

The results of this study confirm that contemporary treatment of TDAVF leads to a high percentage of both angiographic cure and favorable clinical outcomes. Successful treatment is achieved uniformly among ruptured and unruptured lesions, with comparable low morbidity, among endovascular, surgical, and combined-treatment modalities. Endovascular and multimodality approaches appear to be associated with a higher rate of symptom resolution in patients with nonhemorrhagic presentation, compared with standalone surgical approaches. Furthermore, our study reveals a sustained shift from surgery toward an endovascular approach as the preferred treatment technique of TDAVF in the past 2 decades. Advances in the understanding of the pathologic anatomy of TDAVFs coupled with significant improvement in microcatheter and embolic material technology and microsurgical technique are reflected in the achievement of improved neurologic outcomes in this period. Finally, an increasing number of TDAVFs are now diagnosed and treated before they present with hemorrhage, presumably due to wide availability and improved resolution of noninvasive angiography and the recognition of venous hypertension as a prominent cause of neurologic morbidity.

Dural arteriovenous fistulas of the tentorium have been traditionally considered among the most likely to present with hemorrhage or aggressive nonhemorrhagic neurologic dysfunction. In their review of 337 DAVF cases in 1990, Awad et $\mathrm{al}^{10}$ found that $97 \%$ of TDAVFs presented with either hemorrhage or symptomatic venous hypertension. In fact, the overwhelming majority of patients with DAVFs who present with hemorrhage have TDAVFs. ${ }^{5}$ It is now recognized that the presence of retrograde leptomeningeal drainage, not DAVF location, is the major determining factor of the mode of clinical presentation, and TDAVFs are universally associated with retrograde leptomeningeal drainage. ${ }^{11}$ The natural history of symptom- 


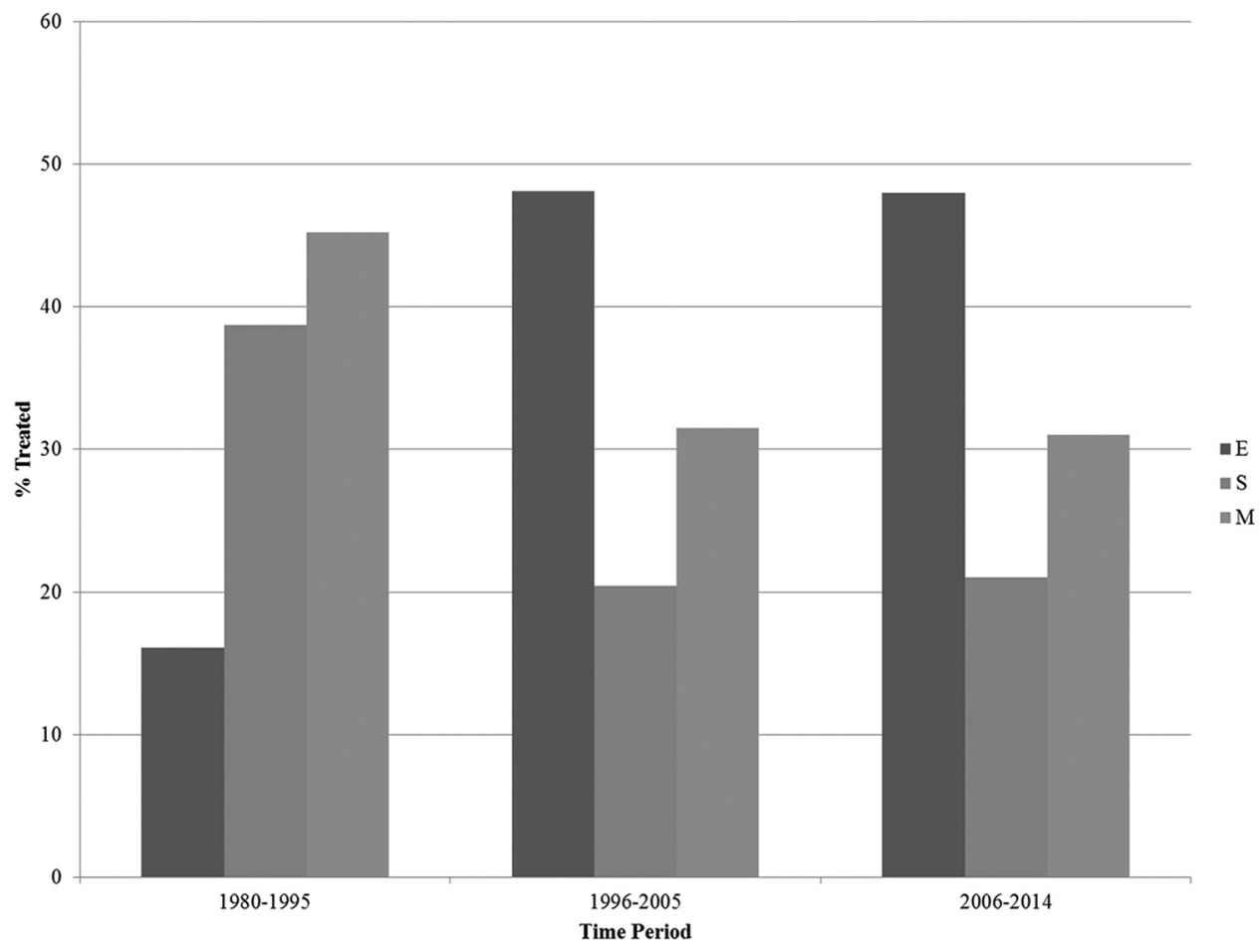

FIG 2. Treatment technique type by time period. E indicates endovascular; S, surgical; $M$, combined.

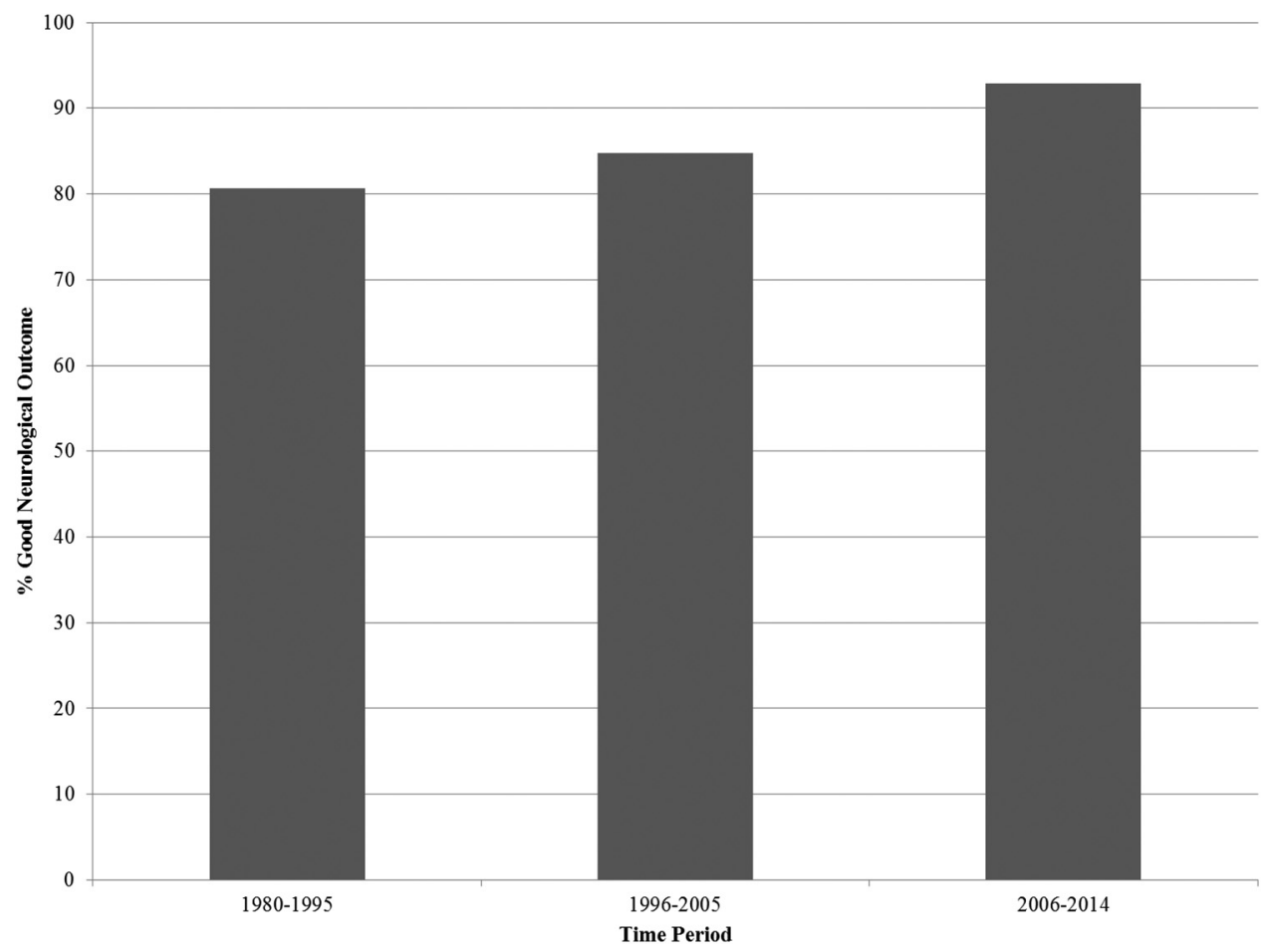

FIG 3. Rate of good neurologic outcome by time period.

atic DAVF with retrograde leptomeningeal drainage is particularly unfavorable. In 1 study of 20 patients who received either no or partial treatment for DAVFs with retrograde leptomeningeal drainage, the annual mortality rate was calculated at $10.4 \%$ and the annual risk for hemorrhage or nonhemorrhagic neurologic deficits after the first presentation were $8.1 \%$ and $6.9 \%$, respectively. Five patients in this series had DAVFs of the tentorium, of whom 3 died and 1 had mod- erate disability on follow-up. ${ }^{12}$ On the other hand, recent studies have shown that asymptomatic DAVFs with retrograde leptomeningeal drainage may have a relatively benign natural course. Careful inspection reveals that this may not necessarily be valid in the case of TDAVFs, as evidenced in a series of 17 asymptomatic patients, in which only 1 patient had a DAVF of the tentorium and who, in fact, presented with subarachnoid hemorrhage after partial treatment. ${ }^{13}$ 


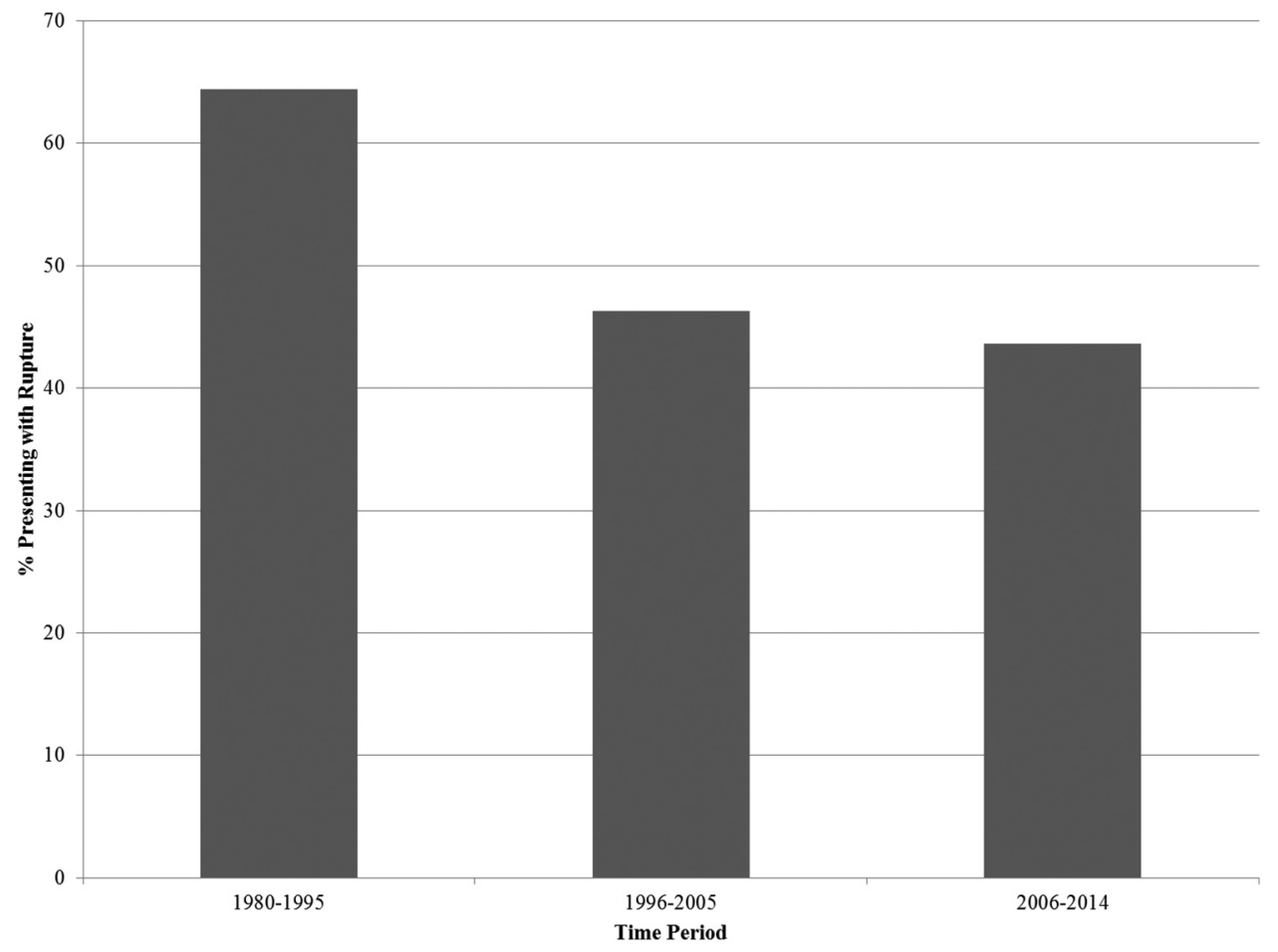

FIG 4. Proportion of patients presenting with ruptured TDAVFs by time period.

Until recently, DAVFs of the tentorium were considered candidates only for surgical interruption in the form of microsurgical disconnection of the proximal leptomeningeal venous drainage. ${ }^{14}$ The introduction of ethylene-vinyl alcohol (Onyx; Covidien, Irvine, California), a permanent, nonadhesive, liquid embolic agent, has revolutionized DAVF treatment and may be the leading cause of the shift toward a predominantly endovascular treatment paradigm in TDAVFs. In transarterial DAVF Onyx embolization, a proximal plug is created around the distal tip of the microcatheter to produce sufficient proximal flow arrest and allow distal penetration of ethylene-vinyl alcohol into the proximal venous outlet while allowing retrograde occlusion of contributing arterial feeders. More recently, dual-lumen balloon microcatheters are used that allow the unopposed forward penetration of Onyx, without the time-consuming effort of plug formation, while concurrently decreasing the risk of premature proximal reflux. ${ }^{15}$ The posterior branch of the middle meningeal artery almost always provides arterial supply to a TDAVF and provides a natural direct access to the venous collector system of the fistula. Furthermore, the middle meningeal artery and its branches run a relatively straight course and are anchored on the dura, making failed microcatheter retrieval rare despite substantial Onyx reflux. Although proximal Onyx reflux may occur with a high margin of safety in the middle meningeal artery, reflux should not be allowed in proximity to the level of the foramen spinosum to avoid inadvertent compromise of the arterial supply to the trigeminal and facial nerves. ${ }^{16}$

Even though we have attempted to summarize data on all published series of treated TDAVFs, our study has certain limitations. The results of separate case series were published by multiple authors who reported independently assessed data and who determined different specific variables that are included in this article. However, the extracted data have been derived from a fairly ho- mogeneous and strictly defined patient population. Furthermore, our study has publication bias, because patients who had either uneventful or poor outcomes may have been excluded from published results. Moreover, treatment modalities have varied during the time course of the published series; this variation makes standardization of treatment paradigms difficult. Last, uniform assessment and reporting of complications in a standardized fashion were lacking. On the basis of the Grading of Recommendations, Assessment, Development and Evaluation framework, the quality of evidence (confidence in estimates) is very low because of imprecision, heterogeneity, and methodologic limitations of the included studies. ${ }^{17,18}$ Nevertheless, this meta-analysis provides useful data to share with patients and families when assessing the risks of treatment of TDAVFs and represents a benchmark against which future studies can be compared. With analysis of $>270$ patients, this is currently the largest study examining outcomes of surgical and endovascular treatment of TDAVFs, to our knowledge.

\section{CONCLUSIONS}

During the past 35 years, patients with TDAVFs have been increasingly presenting with unruptured lesions, receiving endovascular treatment, and experiencing higher rates of good neurologic outcomes. Endovascular treatment was associated with the best long-term neurologic outcomes but lower occlusion rates. Combined endovascular and surgical treatment was associated with high occlusion rates and low morbidity. Increasing use of Onyx may continue to shift the balance more and more toward increasing endovascular treatment as the sole mode of therapy.

Disclosures: Giuseppe Lanzino—UNRELATED: Consultancy: Covidien/ev3.* *Money paid to the institution. 


\section{REFERENCES}

1. Hiramatsu M, Sugiu K, Hishikawa T, et al. Epidemiology of dural arteriovenous fistula in Japan: analysis of Japanese Registry of Neuroendovascular Therapy (JR-NET2). Neurol Med Chir (Tokyo) 2014;54:63-71

2. Söderman M, Pavic L, Edner G, et al. Natural history of dural arteriovenous shunts. Stroke 2008;39:1735-39

3. Byrne JV, Garcia M. Tentorial dural fistulas: endovascular management and description of the medial dural-tentorial branch of the superior cerebellar artery. AJNR Am J Neuroradiol 2013;34:1798-804

4. Cha KC, Yeon JY, Kim GH, et al. Clinical and angiographic results of patients with dural arteriovenous fistula. J Clin Neurosci 2013;20:536-42

5. Daniels DJ, Vellimana AK, Zipfel GJ, et al. Intracranial hemorrhage from dural arteriovenous fistulas: clinical features and outcome. Neurosurg Focus 2013;34:E15

6. Zhou LF, Chen L, Song DL, et al. Tentorial dural arteriovenous fistulas. Surg Neurol 2007;67:472-81; discussion 481-82

7. Yang HC, Kano H, Kondziolka D, et al. Stereotactic radiosurgery with or without embolization for intracranial dural arteriovenous fistulas. Neurosurgery 2010;67:1276-83; discussion 1284-85

8. DerSimonian R, Laird N. Meta-analysis in clinical trials. Control Clin Trials 1986;7:177-88

9. Higgins JP, Thompson SG, Deeks JJ, et al. Measuring inconsistency in meta-analyses. $B M J$ 2003;327:557-60

10. Awad IA, Little JR, Akarawi WP, et al. Intracranial dural arteriovenous malformations: factors predisposing to an aggressive neurological course. J Neurosurg 1990;72:839-50

11. Davies MA, TerBrugge K, Willinsky R, et al. The validity of classification for the clinical presentation of intracranial dural arteriovenous fistulas. J Neurosurg 1996;85:830-37

12. van Dijk JM, terBrugge KG, Willinsky RA, et al. Clinical course of cranial dural arteriovenous fistulas with long-term persistent cortical venous reflux. Stroke 2002;33:1233-36

13. Strom RG, Botros JA, Refai D, et al. Cranial dural arteriovenous fistulae: asymptomatic cortical venous drainage portends less aggressive clinical course. Neurosurgery 2009;64:241-47

14. Thompson BG, Doppman JL, Oldfield EH. Treatment of cranial dural arteriovenous fistulae by interruption of leptomeningeal venous drainage. J Neurosurg 1994;80:617-23

15. Rammos S, Bortolotti C, Lanzino G. Endovascular management of intracranial dural arteriovenous fistulae. Neurosurg Clin $N$ Am 2014;25:539-49

16. Puffer RC, Daniels DJ, Kallmes DF, et al. Curative Onyx embolization of tentorial dural arteriovenous fistulas. Neurosurg Focus 2012;32:E4

17. Balshem H, Helfand M, Schünemann HJ, et al. GRADE guidelines: 3 . Rating the quality of evidence. J Clin Epidemiol 2011;64:401-06

18. Guyatt GH, Oxman AD, Kunz R, et al. GRADE guidelines 6. Rating the quality of evidence-imprecision. J Clin Epidemiol 2011;64:1283-93

19. Abud TG, Nguyen A, Saint-Maurice JP, et al. The use of Onyx in different types of intracranial dural arteriovenous fistula. AJNR Am J Neuroradiol 2011;32:2185-91

20. Bret P, Salzmann M, Bascoulergue Y, et al. Dural arteriovenous fistula of the posterior fossa draining into the spinal medullary veins: an unusual cause of myelopathy — case report. Neurosurgery 1994; 35:965-68; discussion 968-99

21. Fardoun R, Adam Y, Mercier P, et al. Tentorial arteriovenous malformation presenting as an intracerebral hematoma: case report. J Neurosurg 1981;55:976-78

22. Grisoli F, Vincentelli F, Fuchs S, et al. Surgical treatment of tentorial arteriovenous malformations draining into the subarachnoid space: report of four cases. J Neurosurg 1984;60:1059-66

23. Gross BA, Du R. Surgical treatment of high grade dural arteriovenous fistulae. J Clin Neurosci 2013;20:1527-32

24. Hatano T, Bozinov O, Burkhardt JK, et al. Surgical treatment of tentorial dural arteriovenous fistulae located around the tentorial incisura. Neurosurg Rev 2013;36:429-35

25. Huang Q, Xu Y, Hong B, et al. Use of Onyx in the management of tentorial dural arteriovenous fistulae. Neurosurgery 2009;65:28792; discussion 292-93

26. Ito $\mathrm{M}$, Sonokawa $\mathrm{T}$, Mishina $\mathrm{H}$, et al. Reversible dural arteriovenous malformation-induced venous ischemia as a cause of dementia: treatment by surgical occlusion of draining dural sinus-case report. Neurosurgery 1995;37:1187-91; discussion 1191-92

27. Kakarla UK, Deshmukh VR, Zabramski JM, et al. Surgical treatment of high-risk intracranial dural arteriovenous fistulae: clinical outcomes and avoidance of complications. Neurosurgery 2007;61:44757; discussion 457-59

28. Lawton MT, Sanchez-Mejia RO, Pham D, et al. Tentorial dural arteriovenous fistulae: operative strategies and microsurgical results for six types. Neurosurgery 2008;62(3 suppl 1):110-24

29. Lewis AI, Tomsick TA, Tew JM, Jr. Management of tentorial dural arteriovenous malformations: transarterial embolization combined with stereotactic radiation or surgery. J Neurosurg 1994;81:851-59

30. Lewis AI, Rosenblatt SS, Tew JM Jr. Surgical management of deepseated dural arteriovenous malformations. J Neurosurg 1997;87: 198-206

31. Liu C, Xu B, Song D, et al. Clinical approach of using Onyx via transarterial access in treating tentorial dural arteriovenous fistula. Neurol Res 2014;36:983-91

32. Lucas Cde P, Prandini MN, Caldas JG. Analysis of the best therapeutic alternative for intracranial dural arteriovenous malformations. Arq Neuropsiquiatr 2005;63:605-13

33. Lucas CP, Zabramski JM, Spetzler RF, et al. Treatment for intracranial dural arteriovenous malformations: a meta-analysis from the English language literature. Neurosurgery 1997;40:1119-30; discussion $1130-32$

34. Natarajan SK, Ghodke B, Kim LJ, et al. Multimodality treatment of intracranial dural arteriovenous fistulas in the Onyx era: a single center experience. World Neurosurg 2010;73:365-79

35. Ott D, Bien S, Krasznai L. Embolization of a tentorial dural arteriovenous fistula presenting as atypical trigeminal neuralgia. Headache 1993;33:503-08

36. Pandey P, Steinberg GK, Westbroek EM, et al. Intraoperative angiography for cranial dural arteriovenous fistula. AJNR Am J Neuroradiol 2011;32:1091-95

37. Pierot L, Chiras J, Meder JF, et al. Dural arteriovenous-fistulas of the posterior fossa draining into subarachnoid veins. AJNR Am J Neuroradiol 1992;13:315-23

38. Piippo A, Niemela M, van Popta J, et al. Characteristics and longterm outcome of 251 patients with dural arteriovenous fistulas in a defined population. J Neurosurg 2013;118:923-34

39. Rabinov JD, Yoo AJ, Ogilvy CS, et al. ONYX versus n-BCA for embolization of cranial dural arteriovenous fistulas. J Neurointerv Surg 2013;5:306-10

40. Tomak PR, Cloft HJ, Kaga A, et al. Evolution of the management of tentorial dural arteriovenous malformations. Neurosurgery 2003; 52:750-60; discussion 760-62

41. van Lindert E, Hassler W, Kühne D, et al. Combined endovascularmicrosurgical treatment of tentorial-incisural dural arteriovenous malformations: report of five cases. Minim Invasive Neurosurg 2000;43:138-43

42. van Rooij WJ, Sluzewski M, Beute GN. Tentorial artery embolization in tentorial dural arteriovenous fistulas. Neuroradiology 2006;48:737-43

43. Wachter D, Hans F, Psychogios MN, et al. Microsurgery can cure most intracranial dural arteriovenous fistulae of the sinus and nonsinus type. Neurosurg Rev 2011;34:337-45; discussion 345

44. Wajnberg E, Spilberg G, Rezende MT, et al. Endovascular treatment of tentorial dural arteriovenous fistulae. Interv Neuroradiol 2012;18: $60-68$ 